\title{
Reactivity of free radical intermediates that form spontaneously during molecular chlorine action on acetylene and vinyl monomers at low temperatures
}

\author{
D.A. Gordon, V.A. Volodina, and A.I. Mikhailov \\ Institute of Problems of Chemical Physics, Russian Academy of Sciences \\ 1 Semenov Ave., Chernogolovka 142432, Russia \\ E-mail: diliarag@gmail.com
}

Received 24 September, 2012

\begin{abstract}
In this study ESR, UV, VIS and IR spectroscopy, chromatography, calorimetry, and elemental analysis were employed to show the spontaneous formation of free radicals under low-temperature action of molecular chlorine on acetylene monomer $p$-diethynylbenzene, and vinyl monomer acrylamide. These radicals are able to initiate chain reaction of polymerization of monomers at low temperatures. This reaction results in a completely soluble polymer with a yield of $25 \%$, while an insoluble cross-linked polymer has been obtained during polymerization of $p$-diethynylbenzene initiated by other means. For example, under radiolysis by $1000 \mathrm{kGy}$ the soluble fraction of obtained polymer was $\approx 1 \%$ while the overall yield was $\approx 10 \%$. $p$-Diethynylbenzene polymerizes at temperatures close to chlorine melting $(170 \mathrm{~K})$. Polymerization of acrylamide takes place in the temperature range of $180-210 \mathrm{~K}$ with an yield of polymer $\approx 10 \%$. The low-temperature chlorination of a $20 \%$-solution of acrylamide in glycerol enables a twofold increase of the polymer yield, and a tenfold decrease of the chlorine content in it.
\end{abstract}

PACS: 82.35.Cd Conducting polymers;

61.80.Ed $\gamma$-ray effects.

Keywords: free radicals, low-temperature halogenation, low-temperature radical polymerization, polymers with conjugated bonds.

\section{Introduction}

In Refs. 1-3 the spontaneous formation of active free radicals in the reactions of low-temperature halogenation of vinyl monomers, natural and synthetic polymers at 50-170 K has been found. At pressures of $\mathrm{F}_{2}, \mathrm{Cl}_{2}<100$ Torr the concentration of radicals reached values of $10^{17}-10^{20} \mathrm{spin} / \mathrm{g}$, for which, for example, in the radiolysis would require $10^{3}$ or $10^{4} \mathrm{kGy}$. Usually, in order to obtain free radicals, a high energy impact on the system is necessary (i.e., heating, radiation, mechanical, etc.).

It was suggested [2,3] that formation of radicals in the course of low-temperature halogenation occurs in multimolecular complexes from breaking chemical bonds without external energy impact. For molecular halogenation it is sufficient to merely regroup the original bonds in multimolecular complexes and the process takes place without a final break of chemical bonds and the formation of free radicals and ions [4]. Formation of free radicals needs more energy and requires involvement of a larger number of halogen molecules in the complex. Therefore, in the chlorination of vinyl monomer with a double bond opening, for the enthalpy to be negative, it is necessary that the number of chlorine molecules is equal to two or more:

$$
-\underset{\mathrm{H}}{\mathrm{C}}=\underset{\mathrm{H}}{\mathrm{C}}-+n \mathrm{Cl}_{2} \rightarrow \mathrm{R}^{\bullet} \mathrm{Cl}_{k}+m \mathrm{HCl}+\Delta H,
$$

$\Delta H<0$ for $n \geq 2$.

Formed radicals can play an important role in the processes which take place during low-temperature halogenation of monomers and polymers. From the viewpoint of practical application this study addresses the specificity of low-temperature chemical processes. For example, during chlorination of lignocellulose materials, chlorination of the aromatic nucleus (accompanied by formation of toxic chlorphenols and chlorodioxines) can be avoided and only double bonds of the chain can be selectively chlorinated. Prior to this study, the ability of radicals formed during low-temperature halogenation to initiate low-temperature 
polymerization were not available in the literature. Specificity (peculiarities) of halogenation at low-temperatures can allow production of polymers with new characteristics.

The purpose of this study was to investigate the possibility of spontaneous formation of free radicals in the course of molecular chlorine effect to acetylene monomer, paradietinilbenzene ( $p$-DEB), and vinyl monomer, acrylamide (AA), at low temperatures and their ability to initiate polymerization of these monomers. In the case of positive results, the aim was to determine the temperature range of polymerization occurrence and to optimize the process by conducting low-temperature chlorination of AA solutions in ethanol and glycerol.

\section{Experimental}

Ultraviolet, visible, IR and ESR spectroscopy, calorimetry, elemental analysis, chromatography, and viscosimetry methods have been used for investigations. Commercial acrylamide $\left(T_{m}=357.5 \mathrm{~K}\right.$, "Fluka", main substance content $90 \%)$ and $p$-DEB $\left(T_{m}=363 \mathrm{~K}\right.$, content of main substance $99 \%$ ) were purified by reprecipitation from benzene and ethanol solutions, respectively. Chlorine is prepared in the laboratory by the reaction:

$$
2 \mathrm{KMnO}_{4}+16 \mathrm{HCl} \rightarrow 2 \mathrm{KCl}+2 \mathrm{MnCl}_{2}+8 \mathrm{H}_{2} \mathrm{O}+5 \mathrm{Cl}_{2} \text {. }
$$

Apart from carrying out the common etching of chlorine the impurities were also removed at cryogenic conditions. In the course of heating of chlorine samples obtained and frozen at $77 \mathrm{~K}$ the temperature dependence of the vapor pressure has been measured. This dependence was compared with the vapor pressure of pure $\mathrm{HCl}$ and $\mathrm{Cl}_{2}$ vs. temperature [5]. Repeated removal under vacuum of vapor of $\mathrm{Cl}_{2}$ with impurities in the temperature range 120-194 K, if there were mismatches between the vapor pressure values, allowed us to obtain pure chlorine.

The monomer samples (the weights ranging 300-500 mg) were first vacuumized and cooled down to $77 \mathrm{~K}$ then filled with chlorine. The content of the latter in the initial samples ranged from 5 to $50 \mathrm{wt} \%$. In order to increase the contact surface of the reactants in the case of acrylamide solution in ethanol or glycerol, samples were prepared as follows. Solutions were dripped into a vessel containing liquid nitrogen, the resulting frozen balls were ground and after removal of the liquid nitrogen a chlorine sample was deposited on them at $77 \mathrm{~K}$. The process of chlorination of $p$-DEB, AA and solutions of the latter in ethanol or in glycerol was done at the lowest temperature, where it still proceeds at a measurable speed. Ampoules containing monomers, either $20 \%$ solutions of AA in ethanol or glycerol and deposited on them at $77 \mathrm{~K}$ chlorine $\left(T_{m}(\mathrm{Cl})=172 \mathrm{~K}, T_{b}(\mathrm{Cl})=238.4 \mathrm{~K}\right)$ were immersed in a Dewar vessel filled with ethyl alcohol cooled to $110 \mathrm{~K}$ (ethanol devitrification temperature $T_{g}=$ $=110 \mathrm{~K}$ ) and gradually warmed up to $180-210 \mathrm{~K}$ for mo- nomers and to $230 \mathrm{~K}$ for solutions at a rate of $0.5 \mathrm{deg} / \mathrm{min}$ and kept at this final temperatures for 4-5 h. For calorimetric studies carried out as described in Ref. 6 diathermic calorimeter has been used. The output of non-volatile products formed during low-temperature operation of molecular chlorine was determined at $300 \mathrm{~K}$ gravimetrically after removal of unreacted components by evacuation and extraction with ethanol in the case of AA (acrylamide is highly soluble in ethanol, whereas polyacrylamide is not). The insoluble were dried. Molecular weights of the products obtained were determined by either gas chromatography or viscosimetry. Elemental analysis was performed with Pregl's method. The optical absorption spectra in UV, VIS regions were recorded on a spectrometer "Shimadzu UV-3101 PC", the infrared spectra, on a "Perkin Elmer" spectrometer Lambda EZ 210 at $300 \mathrm{~K}$. The ESR spectra were registered on a radiospectrometer 3-cm-band ESR-21 at $77 \mathrm{~K}$ and the microwave field $\sim 10^{-5} \mathrm{~W}$.

\section{Discussion of results}

It is known that under UV or $\gamma$ irradiation, as well as catalytic and thermal action, acetylenic monomers FA and $p$-DEB, polymerize to form chains with conjugated bonds [7-17]. Therefore, the resulting polymers, depending on the length of conjugation, have color from light brown to black, which allows their studies by means of optical spectroscopy $[9,11,15]$.

During low-temperature operation of molecular chlorine on the $p$-DEB, non-volatile viscous dark yellow products were obtained, the viscosity and color of which depend on the time of action of chlorine: the viscosity increases with time, while the color becomes brown. After ten repetitions of the heating from 100 to $210 \mathrm{~K}$, nonvolatile products that are well soluble in ethanol were formed. The monomer conversion was about $15 \%$, the chlorine content in obtained product was $50 \mathrm{wt} \%$. After 20 repetitions of heating, in addition of viscous products, solids insoluble in ethanol but completely soluble in dimethylformamide (DMFA) and tetrahydrofuran (THF) were formed. Low-temperature action of chlorine on $p$-DEB resulted in non-volatile products, completely soluble in DMFA and THF. Generally, the maximum yield of nonvolatiles (viscous and solid), obtained by low-temperature effect of chlorine on $p$-DEB, amounted to approximately $25 \%$. According to elemental analysis the chlorine content in them was about of $40 \mathrm{wt} \%$.

Comparative analysis of non-volatile products obtained by low-temperature operation of molecular chlorine on the $p$-DEB with polymers of $p$-DEB obtained by $\gamma$ irradiation has been carried out. Formation mechanism and properties of these polymers are well known (see Refs. 11-14). Molecular masses of non-volatile products obtained at lowtemperature action of chlorine and $\gamma$ irradiation, are shown in Table 1. 
Table 1. Comparison of the molecular masses of non-volatile products obtained under low-temperature action of molecular chlorine and $\gamma$ irradiation. Here $n$ is the number of monomer units in the polymer

\begin{tabular}{c|c|c|c|c}
\hline \hline Sample & $M_{n}$ & $M_{w}$ & $M_{w} / M_{n}$ & $n$ \\
\hline \hline $\mathrm{FA} / \gamma$ & 450 & 950 & 2.11 & $\sim 9$ \\
$\mathrm{FA} / \mathrm{Cl}_{2}$ & 260 & 290 & 1.12 & $\sim 2$ \\
$p-\mathrm{DEB} / \gamma$ & 1490 & 1720 & 1.15 & $\sim 14$ \\
$\begin{array}{c}\text { (soluble in DMF fraction) } \\
p-\mathrm{DEB} / \mathrm{Cl}_{2} \\
20 \text { times heated } \\
p \text {-DEB/Cl }\end{array}$ & 440 & 1650 & 3.75 & $8-9$ \\
10 times the heating & 330 & 460 & 1.4 & $2-3$ \\
\hline \hline
\end{tabular}

Our data show that with short times of $\mathrm{Cl}_{2}$ action the oligomers consisting of several monomer units (2-3) and soluble in ethanol are formed. At larger times, as mentioned above, in addition to the viscous fraction soluble in ethanol, solids soluble in DMFA and THF are produced. Medium-weight molecular mass of common products is as high as $\sim 1600$. According to elemental analysis the chlorine content is $\approx 40 \%$, i.e., for each monomer unit of non-volatile products obtained under low-temperature effect of chlorine on $p$-DEB, we have $\sim 2$ atoms of chlorine. Based on these data, the low-temperature treatment of molecular chlorine on $p$-DEB results in fully soluble and non-crosslinked (possibly branched) polymers, whose molecules contain 8-9 monomer units. It should be noted that in the polymerization of $p$-DEB by other methods [7,9,11-14] mostly insoluble, crosslinked polymers are formed. The macromolecular soluble fraction of the polymer obtained under $\gamma$ irradiation of $p$-DEB, consists of about 14 monomer units (see Table 1). The yield of this fraction is $\sim 1 \%$ at a total yield of $\sim 10 \%$ (at $D=$ $=1000 \mathrm{kGy}$ ).

The calorimetric studies were carried out to clarify the question of what is the temperature at which the processes of polymerization and chlorination of the monomer and resulting polymers takes place.

Figure 1 shows calorimetric heating curves of pure $\mathrm{Cl}_{2}$ (1) and a mixture of $p$-DEB $+\mathrm{Cl}_{2}$ (2). During heating of $\mathrm{Cl}_{2}$ only an endothermic peak associated with melting of $\mathrm{Cl}_{2}$ $\left(T_{m}(\mathrm{Cl})=172 \mathrm{~K}\right.$, boiling point of $\left.\mathrm{Cl}_{2} T_{b}(\mathrm{Cl})=238.4 \mathrm{~K}\right)$ at temperatures $\sim 170 \mathrm{~K}$ is observed. Melting of $p$-DEB occurs at $363 \mathrm{~K}$, and in the temperature range $77-300 \mathrm{~K}$ no thermal effects associated with changes in the phase state were observed. Heating of $p$-DEB containing $\mathrm{Cl}_{2}$ ( $26 \mathrm{wt} \%$ ), revealed a heat release. For fine specimens of $p$-DEB (obtained by sublimation of the monomer), the reaction starts at $30 \mathrm{~K}$ below the melting point of chlorine, when the mobility of the $\mathrm{Cl}_{2}$ molecules is sufficient to maintain the process at an appreciable rate. Closer to the melting point of chlorine $(\sim 168 \mathrm{~K})$ one can observe the heat release of "explosive"

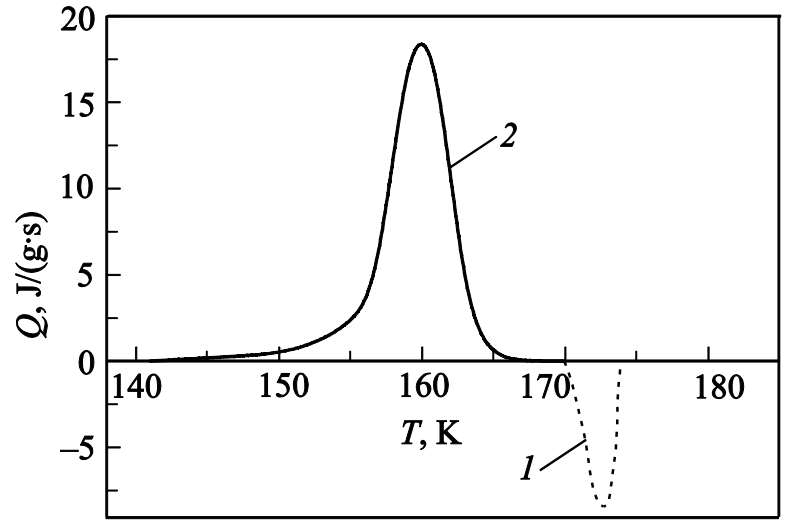

Fig. 1. Heating curves of pure $\mathrm{Cl}_{2}(1)$ and of a mixture $p$-DEB + $+\mathrm{Cl}_{2}(2)$.

nature. Further increase in temperature does not lead to any thermal effect.

To elucidate the mechanism of processes occurring during the low-temperature action of chlorine on $p$-DEB, ESR spectrometry was used. Figure 2 (spectrum 1) shows the ESR spectrum of radicals produced by low-temperature $(77-210 \mathrm{~K})$ action of chlorine on $p$-DEB (registration of the spectra at $77 \mathrm{~K}$ ). The spectrum is a broad singlet with $\Delta H=3 \mathrm{mT}$ and $g=2.003$. While the radicals formed during the interaction of $\mathrm{Cl}_{2}$ with ethylene and methyl methacrylate at low temperatures [2,3], show up as well resolved lines from the hyperfine interaction due to of the unpaired electron with the nuclei of hydrogen and chlorine, in the case of $p$-DEB there is a structureless shaped feature which indicates that delocalization of the unpaired electron takes place along the conjugated chain. Broadening of the singlet line (from conventional 1 to $3 \mathrm{mT}$ ) points to the inclusion of chlorine atoms in the chain. The radicals with measurable rates were formed during the repeated 100 to $210 \mathrm{~K}$ heating of the sample (monomer $+\mathrm{Cl}_{2}$ ) prepared at $77 \mathrm{~K}$. Figure 2 also shows the ESR spectrum of $p$-DEB $\gamma$ irradiated at $300 \mathrm{~K}$ (spectrum 2, registration of spectra at $77 \mathrm{~K}$ ). It is a singlet with $\Delta H=1.2 \mathrm{mT}$ and $g=2.003$ characteristic of polyconjugated systems [11-14]. Thus, under

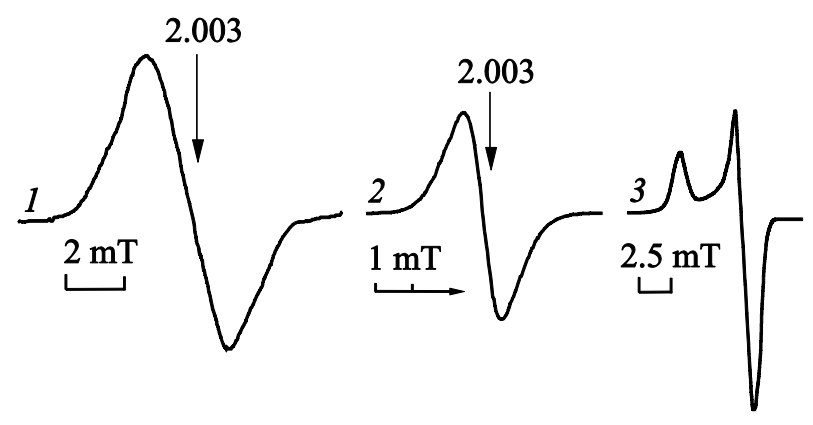

Fig. 2. ESR spectra: radicals produced by low-temperature action of chlorine on $p$-DEB (1), $p$-DEB $\gamma$ irradiated at $300 \mathrm{~K}$ (2), peroxide radical formed during oxygen admission to the system $\mathrm{AA}+\mathrm{Cl}_{2}$ at $170 \mathrm{~K}$ (3). Spectra were recorded at $77 \mathrm{~K}$. 
low-temperature action of molecular chlorine on $p$-DEB, radicals that can initiate polymerization at low temperatures are produced. However, the growing macroradicals loose their activity (which is also observed for other methods used to initiate polymerization of acetylenic monomers), and consequently the resulting polymers have short chains.

It is believed that the reason for the formation of polymers with short chains during free-radical polymerization of acetylenic monomers is the electron delocalization along the chain of conjugation in the growing macroradicals. However, in the low-temperature studies by Gordon et al. [11-14] where it was possible to separate the stages of initiation, growth and termination of chain formation, it was shown that in a linear growing macroradical the electron formed during the opening of a triple bond is located in plane $P_{z}$. This plane is perpendicular to the plane of the conjugated $\pi$-electrons $P_{x y}$ and electron is localized on the terminal fragment of the molecule and therefore is not involved in conjugation. The ESR spectrum is a doublet with $\Delta H=2.6 \mathrm{mT}$. Therefore, a linear growing macroradical must be active. It looses activity when it meets at a polyene chain of the polymer molecule, i.e., a branched polymer is to form. The electron moves to plane $P_{x y}$ and is involved in conjugation, as observed in studies where polymerization was carried out at elevated or normal temperatures when propagating linear macroradicals have the opportunity to meet with neighboring macromolecules. The ESR spectrum for this case is a singlet with $\Delta H=1 \mathrm{mT}$, characteristic of conjugated systems. In Refs. 11, 17 it was suggested that the reason for the low activity of growing linear macroradicals can be also that their growth is tense and thus they start "curling up". Then the active center is shielded, and the macroradical looses its activity. Preservation of the activity of growing linear macroradicals requires their immobilization.

Figure 3 shows optical absorption spectra of monomer $p$-DEB and its polymers produced by gamma irradiation, as well as oligomers and polymers obtained by lowtemperature action of chlorine on the monomers under study. In contrast to the spectra of monomer $p$-DEB (spectrum 1 ) with peaks at $259,271,283$ and $292 \mathrm{~nm}$, the polymers obtained by radiolysis (spectrum 2), show diffuse absorption, running up to $700 \mathrm{~nm}$ with no pronounced absorption peak (the so-called "shoulder") at $~ 365 \mathrm{~nm}$ for the soluble fraction of polidiethynylbenzene, such absorption is typical of polymers with of conjugated bonds $[9,11]$. In the spectra of oligomers and polymers obtained by lowtemperature action of chlorine (spectrum 3), one can also observe the diffuse absorption in the range of 300-450 nm but no absorption band (shoulder). This is apparently because during gamma irradiation the polymer is of uniform composition and the ratio of weight-average molecular mass to number average molecular mass is $M_{w} / M_{n} \sim 1.1$, while for the low-temperature chlorination this ratio is

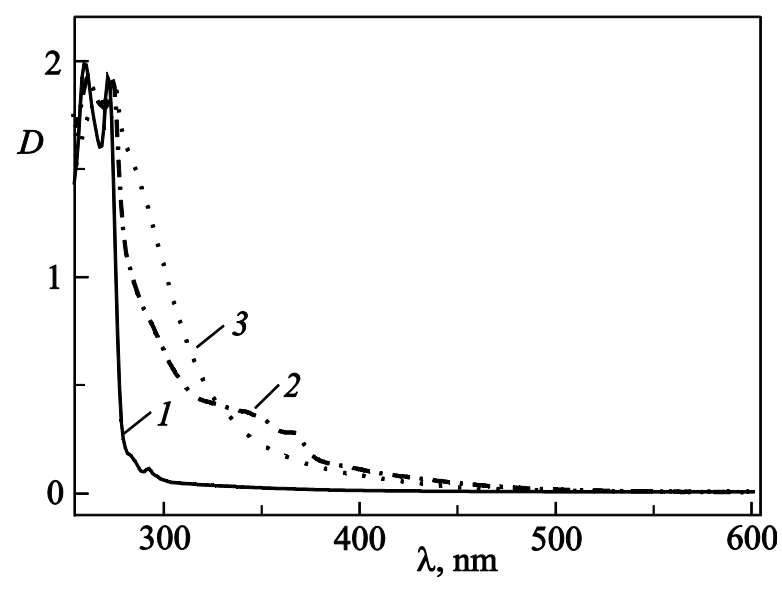

Fig. 3. Optical absorption spectra of monomer p-DEB (1), its polymers obtained by gamma irradiation (2), as well as oligomers and polymers obtained at low-temperature action of chlorine on the $p$-DEB (3).

$M_{w} / M_{n} \sim 3.75$. This means that the polymer, obtained by low-temperature action of chlorine on $p$-DEB, consists of molecules of different lengths, and hence the sites of conjugation can be of different length, which leads to diffuse absorption edge.

Figure 4 shows infrared spectra of $p$-DEB monomers and their oligomers and polymers prepared by lowtemperature action of molecular chlorine and gamma irradiation. Comparing the spectra of monomers and polymers one can see that in polymers, there is a series of bands in the $1600-1750 \mathrm{~cm}^{-1}$ characteristic of polyenes. It is known [18] that in polyenes a few bands appear in this spectral range, sometimes merging into one broad band, and the band shifts toward lower frequencies with increasing number of conjugated bonds. The bands from 1450 to $1600 \mathrm{~cm}^{-1}$, according to the literature [18], is typical of aromatic compounds, and the band at $1580 \mathrm{~cm}^{-1}$ appears only when the benzene ring is associated with an unsaturated group. The appearance of bands in this spectral range in the polymers obtained both by the action of chlorine on $p$-DEB and its radiolysis is evidence of involvement of the benzene ring in the conjugation. This conclusion is consistent with the data obtained in Refs. 12, 13. The bands at 738, 786 and $878 \mathrm{~cm}^{-1}$ are characteristic of the $\mathrm{C}-\mathrm{Cl}$ bond.

Thus, at low temperatures $(100-210 \mathrm{~K})$, the action of molecular chlorine on $p$-DEB is the formation of free radicals, capable of stimulating the reaction of oligomerization and polymerization. The formation of oligomers and polymers with appreciable rates begins near the melting point of chlorine and accelerates the chlorination progresses at melting. In this case, unlike the insoluble crosslinked polymers obtained by other methods of initiation, a spatially non-crosslinked (possibly branched), completely soluble polymers are formed. 

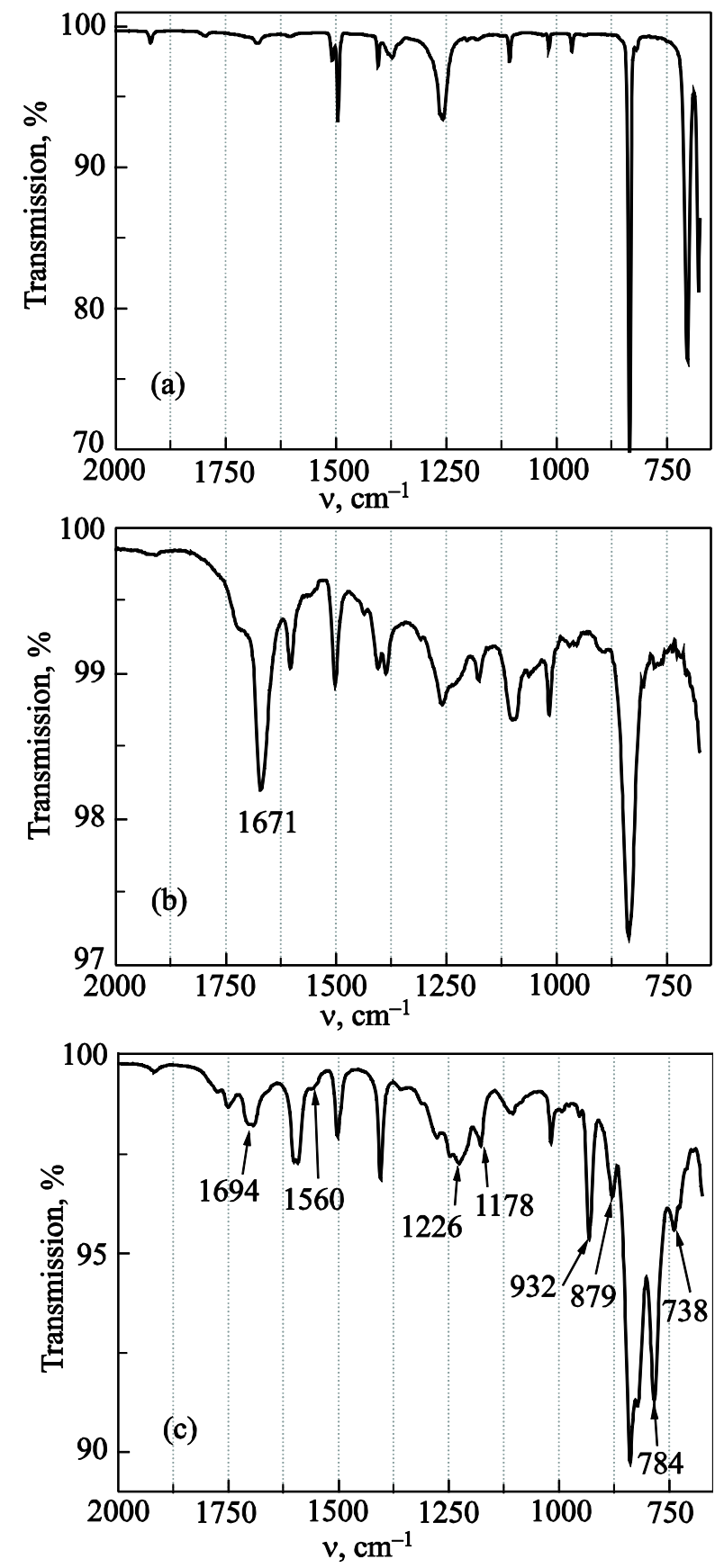

Fig. 4. Infrared spectra of $p$-DEB monomer (a) and its oligomers and polymers prepared by gamma irradiation (b) and lowtemperature operation of molecular chlorine (c).

The product obtained upon low-temperature action of molecular chlorine on acrylamide, after removal of volatile products and products soluble in ethanol, is a powder that changes color from nearly white to gray depending on the chlorine content. Output of non-volatile and insoluble in ethanol product is weakly dependent on the chlorine content in the original sample at contents from 5 to $10 \mathrm{wt} \%$ and amounts to $\sim 8-10 \%$. Gel permeation chromatography (GPC) analysis of the product showed that the bulk of the product has a molecular mass of $(1-2) \cdot 10^{3}$, a high molecu- lar mass $\left(\sim 10^{5}\right)$ fraction being also present, but at a lower proportion (about 1\%). The chlorine content in the product is 10 and $20 \%$ for the chlorine content in the original sample of about 5 and $10 \mathrm{wt} \%$, respectively. It means 4-5 or two molecular units have one atom of chlorine. The chlorine content in the original sample of about $50 \%$ results in a decreased content of insolubles in ethanol products.

Figure 5 shows the infrared spectra of polyacrylamide with a molecular weight of $10^{5}$, obtained by low-temperature radical polymerization under $\gamma$ irradiation of acrylamide as described in Refs. 19, 20 and the products obtained by low-temperature action of molecular chlorine on acrylamide. In both spectra a broad band in the range of $1000-1450 \mathrm{~cm}^{-1}$ with peaks at $1115,1188,1320$, and $1415 \mathrm{~cm}^{-1}$ is observed (in contrast to the spectrum of the monomer). These bands correspond to bands in the polyacrylamide, described in the literature [21,22]. Therefore, on the basis of chromatography and spectroscopy data we can conclude that the unvolatile product insoluble in ethanol and soluble in water obtained during molecular chlorine action on acrylamide is polyacrylamide.

Calorimetric measurements were carried out in order to determine the temperature range of the reactions of polymerization and chlorination of a monomer and a resulting polymer. Figure 6 shows the heating curve of the mixture of AA with molecular chlorine $\left(\mathrm{Cl}_{2}\right.$ content of $\left.10 \mathrm{wt} \%\right)$. One can see the melting peak of chlorine at $172 \mathrm{~K}$ and a heat release in the range of $180-210 \mathrm{~K}$. No thermal effects were observed under further heating. It is natural to associate the heat release at $180-210 \mathrm{~K}$ with polymerization reaction of AA and reactions of chlorine with AA and the resulting polymers, since the calorimetric heating curve of pure AA in the range of investigated temperatures does not show any thermal effects, while the curve for a pure chlorine sample reveals only the peak associated with its melting at $172 \mathrm{~K}$. Thus, we conclude that all processes mentioned above take place in the temperature range of $180-210 \mathrm{~K}$. If all the heat

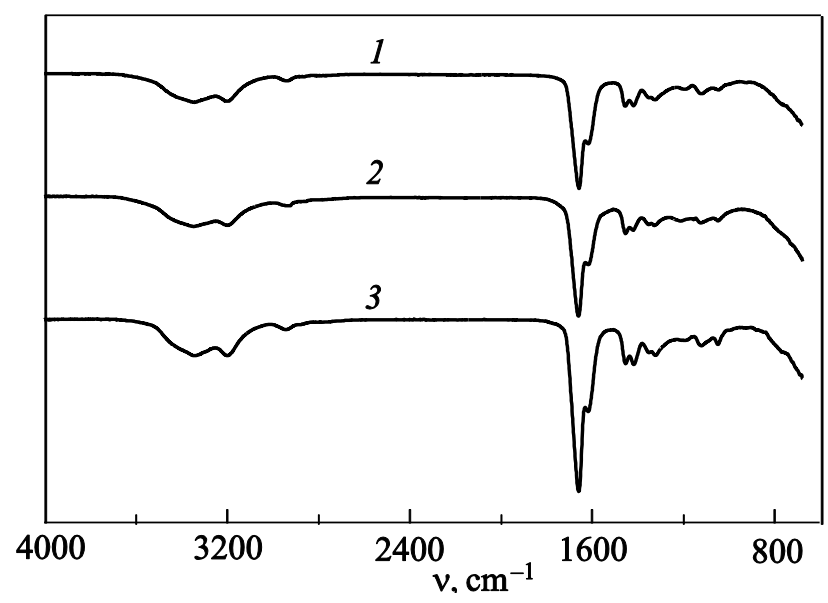

Fig. 5. Infrared spectra of polyacrilamide obtained by gamma irradiation of AA (1), by molecular chlorine action on AA (2), and molecular chlorine action on AA solution in glycerol (3). 


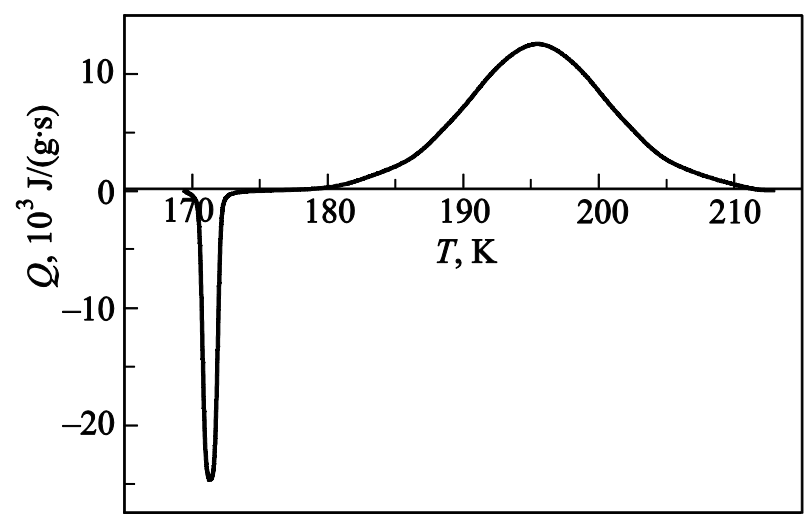

Fig. 6. Heating curve of $\mathrm{AA}+\mathrm{Cl}_{2}$ with $\mathrm{Cl}_{2}$ content of $\sim 10 \mathrm{wt} \%$.

release were attributed to polymerization process, the polymer yield should be $20 \%$. However, the gravimetric yield of the polymer is $10 \%$. This means that the rest of the heat release $(50 \%)$ is due to the chlorination reaction between the monomer and the emerging polymers.

To elucidate the mechanism of processes occurring during low-temperature action of molecular chlorine on acrylamide, ESR studies were carried out. Registration of propagating macroradical, as it was done for the propagating macroradical during low-temperature chlorination of $p$-DEB, failed. However, peroxide radical [16] recorded at admission of oxygen into the system $\mathrm{AA}+\mathrm{Cl}_{2}$ at $170 \mathrm{~K}$ (Fig. 2, spectrum 3 ) is evidence in favor of a radical mechanism of the processes occurring during low-temperature chlorination of acrylamide. It is known [23] that contact of irradiated polymers with oxygen produces peroxide radicals through an interaction between oxygen and radicals formed in polymers during irradiation. It means that these peroxyde radicals are secondary radicals. Therefore, we can conclude that peroxide radical registered in our case is also secondary radicals formed during interaction between oxygen and radicals formed in the course of low-temperature action of molecular chlorine on acrilamide.

The above-mentioned reactions which take place during molecular chlorine action on acrylamide can be represented schematically as follows:
1. Polymerization reaction:

a) formation of radicals $R^{\bullet}$ :<smiles>NC(=O)C=CCC(Cl)(CCl)[C+](N)C(=O)O</smiles>

$$
\mathrm{M}+2 \mathrm{Cl}_{2} \rightarrow \mathrm{R}^{\bullet}+\mathrm{HCl}+\mathrm{Cl}^{\bullet} ; \mathrm{M}+\mathrm{Cl}^{\bullet} \rightarrow \mathrm{R}^{\bullet}
$$

According to Ref. 2, radical formation during lowtemperature effect of molecular chlorine to vinyl monomer without any energy impact may result in the simultaneous reactions in a molecular complex consisting of one monomer molecule and two chlorine molecules. The energy required for simultaneous opening of $\mathrm{C}=\mathrm{C}, \mathrm{C}-\mathrm{H}$ and $\mathrm{Cl}-\mathrm{Cl}$ bonds is compensated by the exothermic effect of chlorine alkyl radical formation, $\mathrm{HCl}$ molecules, and chlorine radical.

b) radicals $R^{\bullet}$ initiate polymerization of monomer:

$$
\mathrm{R}^{\bullet}+\mathrm{M} \rightarrow \mathrm{R}_{p}^{\bullet}
$$

c) reaction of chain propagation:

$$
\mathrm{R}_{p}^{\bullet}+\mathrm{M} \rightarrow \mathrm{R}_{p}^{\bullet}+\mathrm{P}
$$

d) reaction of chain termination:

$$
\mathrm{R}_{p}^{\bullet}+\mathrm{R}_{p}^{\bullet}, \quad \mathrm{Cl}^{\bullet} \rightarrow \mathrm{P}
$$

where $\mathrm{M}$ is the monomer (in this case acrylamide), $\mathrm{R}^{\bullet}$ is the monomeric radical, $\mathrm{R}_{p}^{\bullet}$ is the propagating macroradical, $\mathrm{P}$ is the polymer.

2. Chlorination reaction of the monomer and polymers can be represented as follows:

a) monomer:<smiles>C=C(C(N)=O)[C@H](Cl)[C](Cl)C=C(Cl)[C](Cl)C(Cl)=CC(N)=O</smiles>

b) polymers:

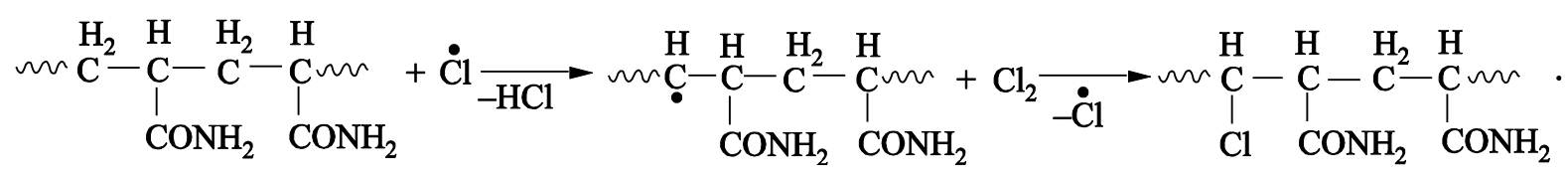

According to Ref. 24 the strength of a bond in $\beta$ position with regard to free radical center is lower by $5-10 \mathrm{kcal} / \mathrm{mol}$ than in $\alpha$ position. It means that chlorination in $\beta$ position is preferable. For a more definite answer to the question of the structure of the polymer (namely, the location of halogen substituent in the polymer chain), we suggest to conduct appropriate NMR studies. Thus, it is shown that the radicals formed during low-temperature action of molecular chlorine 
on AA, initiate the polymerization reaction, which takes place at temperatures $180-210 \mathrm{~K}$ with a yield of polymer of $\approx 9-10 \%$.

However, it is known [19] that there are ways to significantly increase the yield of the polymer in the case of acrylamide low-temperature polymerization. It was found that heating of a $20 \%$-solution of acrylamide in ethyl alcohol $\gamma$ irradiated at $77 \mathrm{~K}$, the polymer yield at a dose of $20 \mathrm{kGy}$ reaches $80 \%$, while $\gamma$ irradiation by the same dose of $20 \mathrm{kGy}$ of crystalline acrylamide leads to a polymer yield of $\sim 3 \%$. The reaction occurs in the temperature range of alcohol solution devitrification (120-130 K). At these temperatures the mobilities of monomer radicals and monomer molecules are sufficient for effective initiation and growth of polymer chains but the mobility of propagating macroradicals is not sufficient for their recombination. Therefore, there is a possibility for living radical polymerization, which results in a product with high yields. In Ref. 25 the polymerization of acrylamide in the matrix of glycerol (polymer yield $\sim 85 \%$ ) was studied. The reaction was initiated by radicals formed in glycerol during storage under day light. The reaction can also be initiated by UV and $\gamma$ irradiation.

Thus, the effect of molecular chlorine (chlorine content in the original sample is $\sim 10 \%$ ) on a $20 \%$ acrylamide solution in ethanol at low temperatures leads to radical polymerization of acrylamide, which proceeds in the temperature range $170-230 \mathrm{~K}$, and the formation of polyacrylamide with a molecular mass of $\sim(1-2) \cdot 10^{3}$. The chlorine content in it, according to the elemental analysis is reduced from 17.7 to $13.5 \%$, compared with the polymer obtained by the chlorination of pure monomer. However, the efficiency of the process remained the same $(\approx 10 \%)$. The radicals formed during low-temperature chlorination of the solutions of acrylamide in glycerol, also initiated polymerization reaction. Polymerization and chlorination reactions take place in the temperature range 200-250 K. Polyacrylamide forms during this process with a yield of $\approx 18 \%$, molecular weight of $\approx 2 \cdot 10^{5}$, and chlorine content of $\sim 2 \%$, the latter being roughly 10 times smaller than in polymers produced by chlorination of pure acrylamide. The increased polymer yield at low-temperature chlorination of acrylamide in glycerols apparently, due to participation in polymerization of the radicals formed in the glycerol under the influence of daylight, as shown in Ref. 25, and with more viscous media than in the case of AA solution in ethanol.

\section{Conclusion}

For the first time it has been shown that during lowtemperature chlorination of $p$-DEB free radicals form which are able to initiate polymerization process. Polymerization takes place close to chlorine melting $(170 \mathrm{~K})$ simultaneously with chlorination process. The resulting polymer with a yield of $25 \%$ is absolutely soluble while the polymer obtained with other methods of polymerization initiation is cross-linked and insoluble. Radicals formed during low-temperature action of molecular chlorine on acrylamide also initiate the polymerization process which proceeds simultaneously with chlorination in the temperature range 180-210 K. Low-temperature action of molecular chlorine on acrylamide + glycerol mixture leads to a two-fold increase of the polymer yield, and in a ten-fold decrease of chlorine content in it.

\section{Acknowledgments}

The authors are grateful to P.S. Mozhayev (IPCP RAS, Chernogolovka) for the treatment of monomers and the synthesis of chlorine, to A.I. Kuzaev (IPCP RAS, Chernogolovka) and to A.N. Ryabev (INEOS, Moscow) for the GPC analysis of the products.

1. G.A. Kapralova, $P h D$ thesis, Moscow Institute of Chemical Physics RAS (1964).

2. S.I. Kuzina, S.V. Demidov, N.N. Denisov, and A.I. Mikhailov, Rus. Chem. Bull. 2, 335 (1999).

3. S.I. Kuzina, A.V. Kulikov, S.V. Demidov, S.V. Moravskii, and A.I. Mikhailov, Rus. J. Phys. Chem. 79, 797 (2005).

4. G.B. Sergeev and V.V. Smirnov, Molecular Halogenation of Olefines, Moscow University Press, Moscow (1985).

5. D.R. Stull, Tables of Individual Compounds Vapor Pressure, Izd-vo Inostr. Literature, Moscow (1949), p. 72.

6. I.M. Barkalov and D.P. Kiryukhin, Int. Rev. Phys. Chem. 13, 337 (1994).

7. A.A. Berlin, Chem. Techn. Polymers 7-8, 139 (1960).

8. I.M. Barkalov, A.I. Berlin, V.I. Gol'danskii, and B.G. Dzantiev, Polymer Sci. A 2, 1103 (1960).

9. D.A. Gordon and A.I. Mikhailov, Fiz. Nizk. Temp. 35, (2009) [Low Temp. Phys. 35, 269 (2009)].

10. Z.S. Kiyashkina, AD. Pomogailo, A.I. Kuzaev, G.V. Lagodzinskaya, and F.S. D'yachkovskii, Polymer Sci. A 21, 1796 (1979).

11. D.A. Gordon and A.I. Mikhaylov, J. Low Temp. Phys. 139, 675 (2005).

12. D.A. Gordon, I.I. Migunova, and A.I. Mikhaylov, Dokl. Akad. Nauk SSSR 213, 368 (1973).

13. D.A. Gordon and A.I. Mikhailov, Polymer Sci. A 17, 1663 (1975).

14. Delyara A. Gordon and Alfa I. Mikhailov, J. Polymer Sci. B. Polymer Phys. 32, 2405 (1994).

15. A.A. Petrus', High Energy Chem. 5, 8, 197 (1974).

16. L.A. Blyumenfeld, V.V. Voevodskii, and A.G. Semenov, The Use of Electron Paramagnetic Resonance in Chemistry, SO AN SSSR, Novosibirsk (1962), p. 241.

17. D.A. Gordon, A.I. Bol'shakov, A.I. Mikhailov, and I.M. Barkalov, Polymer Sci. A 32, 943 (1990).

18. L.A. Kasizina and N.B. Kupletskaya, The Use of $U V, I R$, NMR and Mass-Spectroscopy in Organic Chemistry, Moscow University Press, Moscow (1979). 
19. A.I. Bol'shakov, A.I. Mikhailov, I.M. Barkalov, and V.I. Gol'danskii, Polymer Sci. A 8, 1820 (1978).

20. V.A Volodina, D.A. Gordon, A.I. Bol'shakov, and A.I. Mikhailov, Mendeleev Commun. 16, 270 (2006).

21. E. Pretsch, P. Büllmann, and C. Affolter, Structure Determination of Organic Compounds. Tables of Spectral Data, Springer-Verlag, Berlin, Heidelberg (2000), p. 438.

22. R.F. Colleti, H.S. Gold, and S. Dubrovski, Appl. Spectr. 41, 1185 (1987).
23. S.Ya. Pshezhetzkii, A.G. Kotov, V.K. Milinchuk, V.A. Roginskii, and V.I. Tupikov, Free Radicals ESR in Radiation Chemistry, Moscow (1972), p. 480.

24. E.T. Denisov, Kinetics of Homogeneous Reactions Moscow (1978), p. 367.

25. A.I. Bol'shakov, D.P. Kirukhin, and S.I. Kuzina, Polymer Sci. A 50, 1777 (2008). 